\title{
ESTUDO DA TRANSFERÊNCIA DE MASSA NA DISSOLUÇÃO DE PARTICULAS DE SAL NO ESCOAMENTO DE SALMOURA.
}

\author{
L. A. A. MARTINS, C. M. SCHEID, S. C. MAGALHÃES, e L. A. CALÇADA \\ Universidade Federal Rural do Rio de Janeiro, Departamento de Engenharia Química \\ E-mail para contato calcada@ufrrj.br
}

\begin{abstract}
RESUMO - A perfuração de leitos salinos gera material particulado composto por misturas de sais que tendem a se dissolver em fluidos de perfuração a base de água ao longo da região anular formada entre a parede do poço e a coluna de perfuração. A dissolução pode causar alterações nas propriedades dos fluidos e, consequentemente, ocasionar graves problemas. Objetivou-se com este trabalho, o estudo da dissolução de partículas de sal em salmoura no escoamento axial em dutos. Neste contexto, foi estudado um modelo matemático composto por equações fenomenológicas baseadas nas equações de conservação de massa para as fases sólida e líquida. Uma unidade experimental foi construída para levantar dados necessários para a estimação de parâmetros do modelo matemático. O modelo foi capaz de simular o de dissolução salina em salmouras, apresentando desvios inferiores a 2,3\%.
\end{abstract}

\section{INTRODUÇÃO}

Na perfuração de leitos salinos, cascalhos de sal são gerados na parte inferior da coluna pela ação da broca de perfuração. Estes cascalhos entram na região anular do poço e são transportados para a parte superior da coluna por arraste promovido pela circulação de fluidos de perfuração, e tendem a se dissolverem em fluidos de perfuração a base de água. Essa dissolução pode causar mudanças nas propriedades físico-químicas e reológicas do fluido. A perda de propriedades reológicas do fluido de perfuração, por exemplo, pode gerar acúmulo de cascalhos na parte inferior da coluna, ocasionando problemas operacionais. Outro problema é a dissolução das paredes do poço levando ao enfraquecimento das paredes do mesmo ou lixiviação da formação salina (DURIE \& JESSEN, 1964; AKSEL'RUD et al. 1992).

Na literatura há alguns estudos sobre a dissolução de partículas, porém poucos trabalhos tratam deste fenômeno em um sistema com as características descritas anteriormente. Alguns dos estudos conceitualmente mais próximos têm como foco a lixiviação de sal em cavernas (AKSEL'RUD et al. 1992). O objetivo deste trabalho é estudar a dissolução de partículas de sal no escoamento em processos de perfuração de poços de petróleo. Com este propósito, uma unidade experimental foi construída e perfis de concentração foram levantados e usados como dados de na estimação do coeficiente global de transferência de massa. A partir desta modelagem, foi possível simular a dissolução gerando perfis de concentração da fase líquida (salmoura), temperatura e fração de sólidos em escoamento. 


\section{MATERIAIS E MÉTODOS}

A metodologia empregada para este estudo contempla duas abordagens necessárias para a elaboração de um modelo matemático capaz de representar o processo físico. O fluxograma descrito na figura 1 representa de forma simplificada a metodologia proposta (MARTINS, 2014).

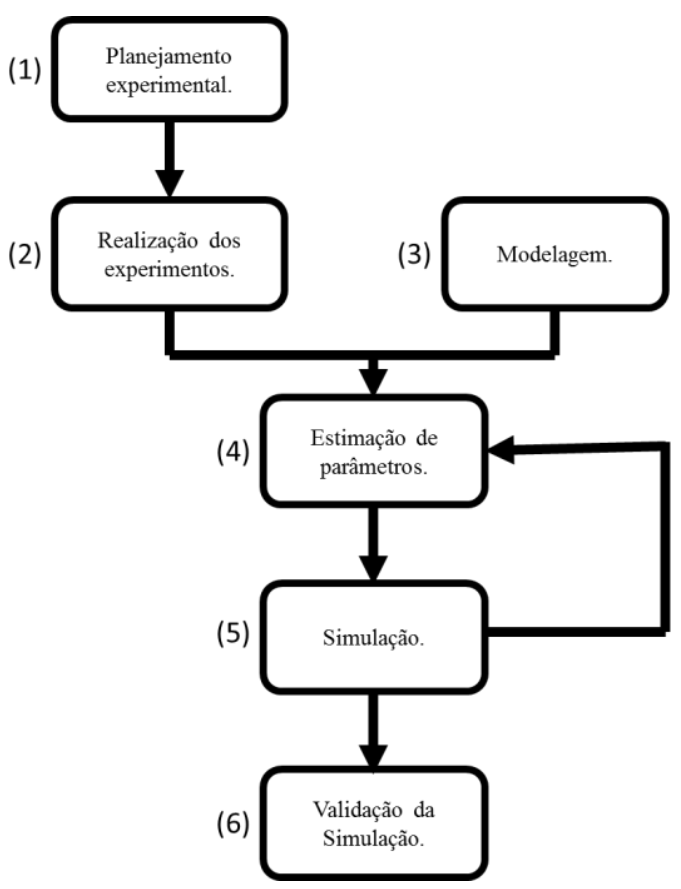

Figura 1- Fluxograma simplificado da metodologia proposta.

O fluxograma de análise desenvolvido começa com a abordagem experimental (1 e 2) e seguido pela abordagem matemática (3). A parte experimental deste estudo tem objetivo de gerar dados de perfil de concentração para estimar o coeficiente global de transferência de massa (K) entre o fluido e partículas sólidas a partir da resolução das equações diferenciais parciais que modelam o processo. A abordagem experimental e matemática permitem a estimação do parâmetro K (4). As curvas simuladas (5) pelo modelo matemático são confrontadas com os resultados experimentais para a estimação de parâmetro K. Se as curvas simuladas não se aproximarem dos valores experimentais a estimação é feita novamente até que sejam obtidos desvios inferiores a $3 \%$. O processo final desta metodologia é a validação (6) deste modelo utilizando dados experimentais não usados para a estimação de parâmetros.

A figura 1 apresenta um esquema da unidade experimental que foi construído para permitir o escoamento de uma mistura de sal particulado e salmoura através de uma calha aberta. 


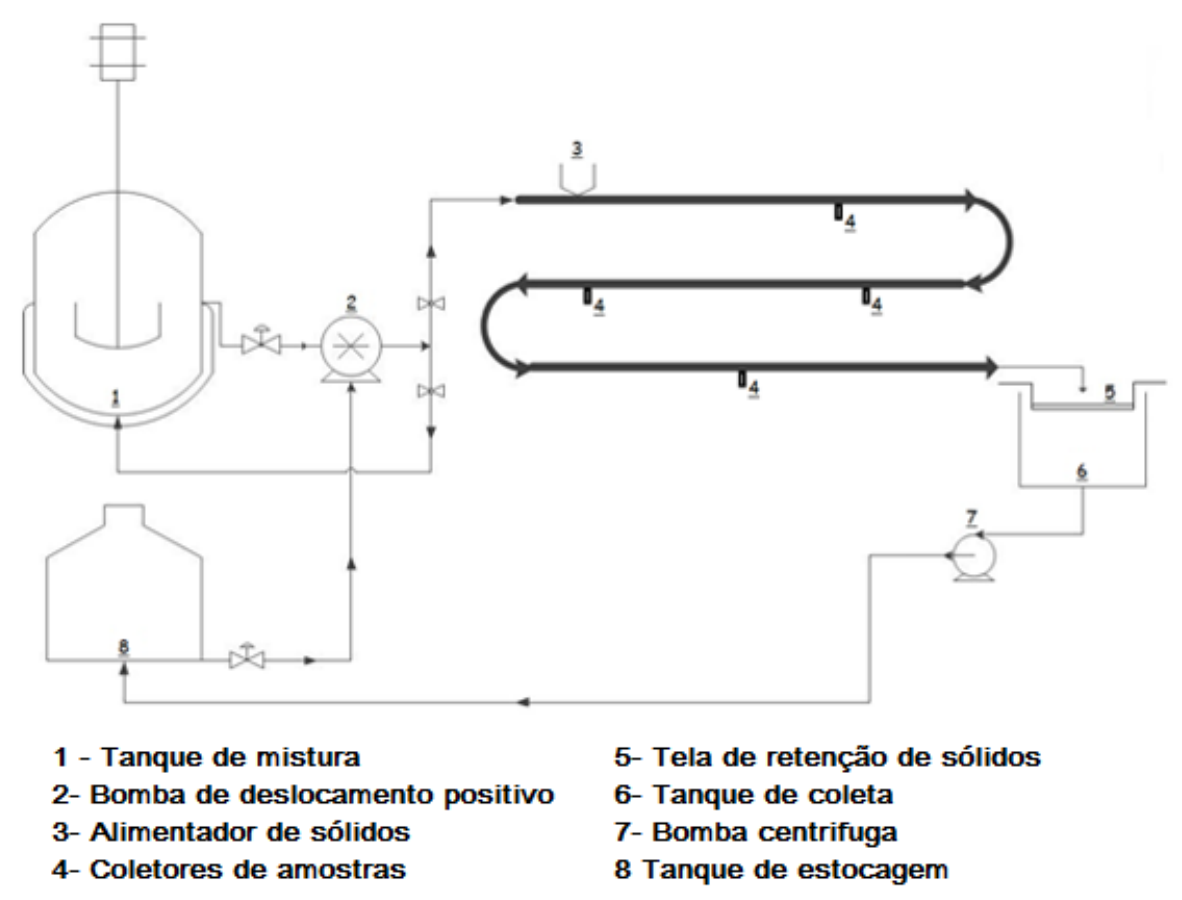

Figura 1- Esquema da unidade experimental de escoamento.

O sal suspenso é carreado pela salmoura ao longo da calha (linha escura mais espessa) onde ocorre a dissolução do sal. Durante o escoamento, amostras são retiradas ao longo deste percurso (4). A unidade experimental é composta por um tanque de mistura de $2000 \mathrm{~L}$ (1). O fluido é bombeado por uma bomba helicoidal (2). A calha é composta por um tubo de PVC de $150 \mathrm{~mm}$, com um comprimento total de $29 \mathrm{~m}$ e uma inclinação de $5^{\circ}$. O tubo é preenchido parcialmente pela mistura durante o escoamento. Um alimentador de sólidos modelo, RD100/75 Retcsh é posicionado possibilitando a alimentação de partículas de sal $(\mathrm{NaCl})$ comercial pela janela aberta no início da calha. Essa unidade também tem um tanque de coleta de 500L (6) logo após o final da calha. Este tanque recebe a mistura de salmoura e sal da calha e uma bomba de $3 / 4 \mathrm{hp}$ bombeia a salmoura para o tanque de estocagem (8). Na entrada do tanque de coleta há uma tela de retenção de sólidos de abertura MESH 100 (5) para a separação do material particulado não solubilizado. O cloreto de sódio foi utilizado por ser o sal predominante em formações do pré-sal. Quatro amostras da fase líquida são coletadas por seringas (4) conectadas a calha nas distâncias 5,5; 13,0; 20,5 e $26,7 \mathrm{~m}$. Com resultado tem-se o perfil de concentração da salmoura ao longo do escoamento e do tempo.

Os experimentos foram planejados para assegurar a repetibilidade experimental, os experimentos foram feitos em triplicata, e permitir a estimação do coeficiente global de transferência de massa com confiança de $95 \%$. A tabela 1 apresenta a malha experimental proposta. Maiores detalhes do trabalho experimental podem ser encontrados em Martins (2014). 
Tabela 1 - Malha experimental em condições de $1 \mathrm{~atm}, 30^{\circ} \mathrm{C}$ e valor experimental da concentração de equilíbrio é de 315,15 g/L.

\begin{tabular}{cccc}
\hline $\mathrm{C}$ & $\mathrm{C}_{0}(\mathrm{~g} / \mathrm{L})$ & $\mathrm{Q}(\mathrm{L} / \mathrm{s})$ & $\mathrm{W}(\mathrm{g} / \mathrm{s})$ \\
\hline 1 & 32,0 & 1,00 & 24 \\
2 & 32,0 & 1,00 & 48 \\
3 & 32,0 & 2,00 & 24 \\
4 & 32,0 & 2,00 & 48 \\
5 & 32,0 & 1,50 & 36 \\
6 & 90 & 1,00 & 24 \\
7 & 90 & 1,00 & 48 \\
8 & 90 & 2,00 & 24 \\
9 & 90 & 2,00 & 48 \\
10 & 90 & 1,50 & 36 \\
11 & 175 & 2,00 & 40 \\
12 & 175 & 2,00 & 30 \\
13 & 175 & 2,00 & 35 \\
14 & 175 & 2,00 & 20 \\
15 & 258 & 2,00 & 40 \\
16 & 258 & 2,00 & 30 \\
17 & 258 & 2,00 & 35 \\
18 & 258 & 2,00 & 20 \\
\hline
\end{tabular}

\section{MODELO MATEMÁTICO}

O modelo matemático utilizado para descrever a dissolução de $\mathrm{NaCl}$ em salmoura é composto por duas equações diferenciais parciais (EDP) obtidas com base na conservação de massa para a fase líquida e sólida (BIRD, et al., 2002 e MAGALHÃES et al., 2011, MARTINS, 2014 E PATRÃO, 2014). A equação 1 foi gerada pelo balanço de massa para o sal em solução e a equação 2 pelo balanço de massa de sal na fase sólida.

As hipóteses adotadas para estas equações diferenciais são:

- Regime transiente;

- Regime plenamente turbulento e adoção de uma velocidade média e constante;

- Suposição de que a velocidade do sólido em transporte é a mesma do fluido em escoamento;

- Sistema isotérmico;

- Concentração de saturação constante;

- Escoamento apenas axial;

- Fluido incompressível;

- Escoamento em duas fases 
- Fase líquida- Solução de água e $\mathrm{NaCl}$;

- Fase sólida- Partículas de $\mathrm{NaCl}$ na forma sólida;

$\frac{\partial}{\partial t} C(z, t)+\bar{v}_{z}\left(\frac{\partial}{\partial z} C(z, t)\right)=K \cdot a \cdot\left(C^{*}-C(z, t)\right)$

C.I., $C(z, 0)=C_{o}$,

C.C., $C(0, t)=C_{o}$.

$\rho_{S}\left(\frac{\partial}{\partial t} \varepsilon_{S}(z, t)+\bar{v}_{z}\left(\frac{\partial}{\partial z} \varepsilon_{S}(z, t)\right)\right)=-K \cdot a \cdot\left(C^{*}-C(z, t)\right)$

C.I.,$\varepsilon_{s}(z, 0)=\varepsilon_{o}$

C.C., $\varepsilon_{s}(0, t)=\varepsilon_{0}$.

O coeficiente $\mathrm{K}$ é um parâmetro a ser estimado utilizando dados experimentais. A área específica de transferência de massa $a$ foi calculado pela equação 3 .

$$
\mathrm{a}=\frac{6 \cdot \varepsilon_{\mathrm{s}}(\mathrm{z}, \mathrm{t})}{\overline{\mathrm{D}_{\mathrm{p}}}},
$$

onde $\overline{\mathrm{D}_{\mathrm{p}}}$ é o diâmetro médio de Sauter da população de partículas salinas (MASSARANI, 2002).O estudo foi feito em condições de escoamento com turbulência plena e considerou-se um valor médio e constante para a velocidade do escoamento. Nesse trabalho, o procedimento para resolução deste sistema de EDP's foi a discretização por diferenças finitas da variável espacial z. Este processo origina um sistema de EAD's no tempo em cada célula de discretização, que foram resolvidas posteriormente pela técnica de Euler. 0 programa simulador e estimador de parâmetros foi implementado em linguagem FORTRAN. A estimação do coeficiente K foi feita usando a técnica da Máxima Verossimilhança baseada no trabalho de Anderson et al. ( 1978). Maiores detalhes do trabalho computacional podem ser encontrados em Martins (2014).

\section{RESULTADOS E DISCUSSÃO}

Os resultados de concentração de salmoura ao longo do escoamento gerados pelo modelo e os respectivos valores experimentais foram apresentados nas figuras $2 \mathrm{a}$ e $2 \mathrm{~b}$. Os perfis de concentração têm crescimento expressivo até $10 \mathrm{~m}$ para a concentração inicial de salmoura de 32 $\mathrm{g} / \mathrm{L}$, figura 2a. Para as outras concentrações de alimentação de salmoura, a concentração aumenta praticamente até o final do sistema. Como exemplo de dado típico foram apresentados dados na figura $2 \mathrm{~b}$ para a concentração de alimentação da salmoura em $258 \mathrm{~g}$. Nas concentrações mais baixas é possível observar um rápido aumento da concentração e posterior estagnação por conta da 
completa dissolução de todas as partículas de sal alimentadas. Em concentrações iniciais de salmoura mais altas, a concentração da salmoura não alcança um valor fixo, principalmente nas figuras 4 e 5. Em concentrações mais elevadas da salmoura, a dissolução é mais lenta devido ao menor potencial cinético da solução, pela aproximação da concentração de saturação. Nota-se que o coeficiente $\mathrm{K}$ é dependente da concentração e tende a ser menor com o aumento da concentração da salmoura, vide figura 3. As simulações para a fração volumétrica de sólidos $(\varepsilon)$ para os dois casos foram apresentados nas figuras $4 \mathrm{a}$ e $4 \mathrm{~b}$. Resultados completos dos dados apresentados neste trabalho podem ser encontrados em Martins (2014) e Patrão (2012).

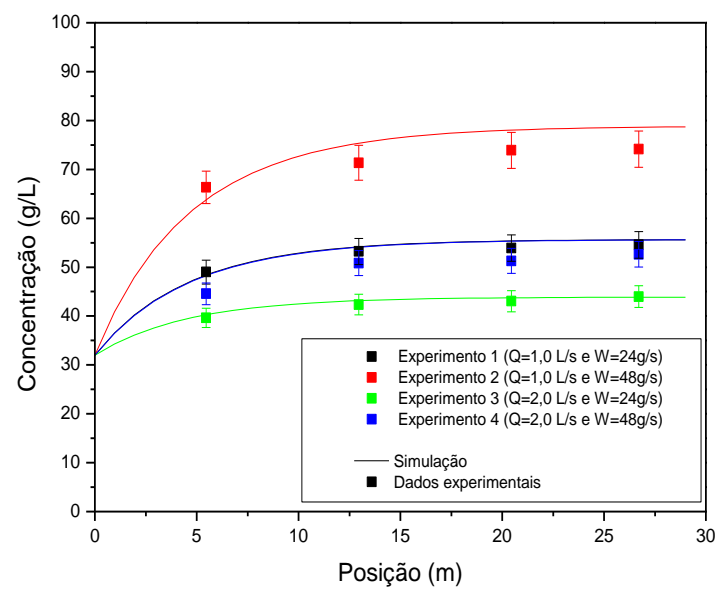

(a)

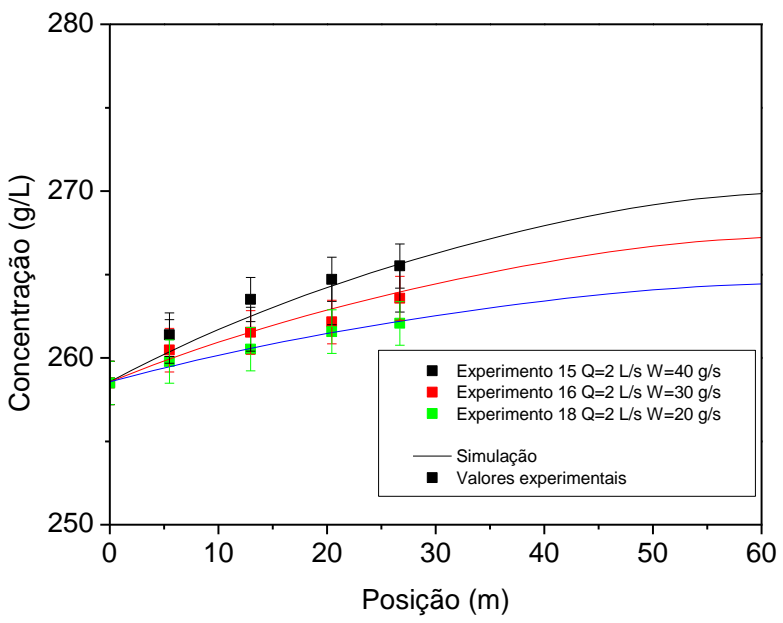

(b)

Figura 2 - Simulações e dados experimentais para concentração em função da posição. Figura 2a dados a $32 \mathrm{~g} / \mathrm{L}$ e Figura $2 \mathrm{~b}$ dados a $258 \mathrm{~g} / \mathrm{L}$.

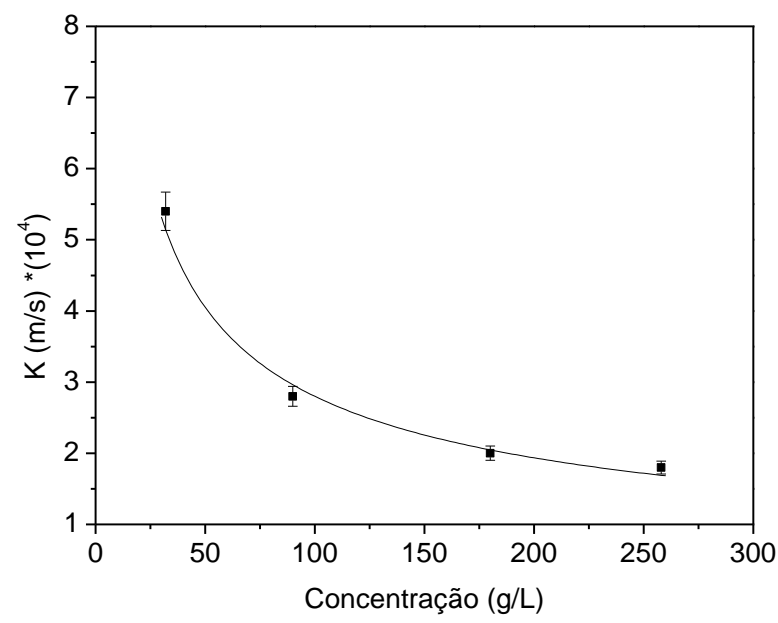

Figura 3 - K em função da concentração da fase líquida. 


\section{9 a 22 de outubro de 2014 \\ Florianópolis/SC}

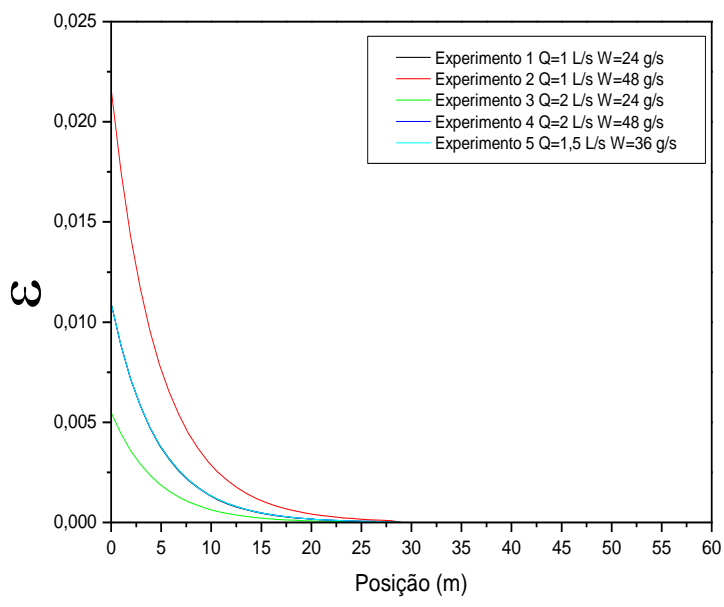

(a)

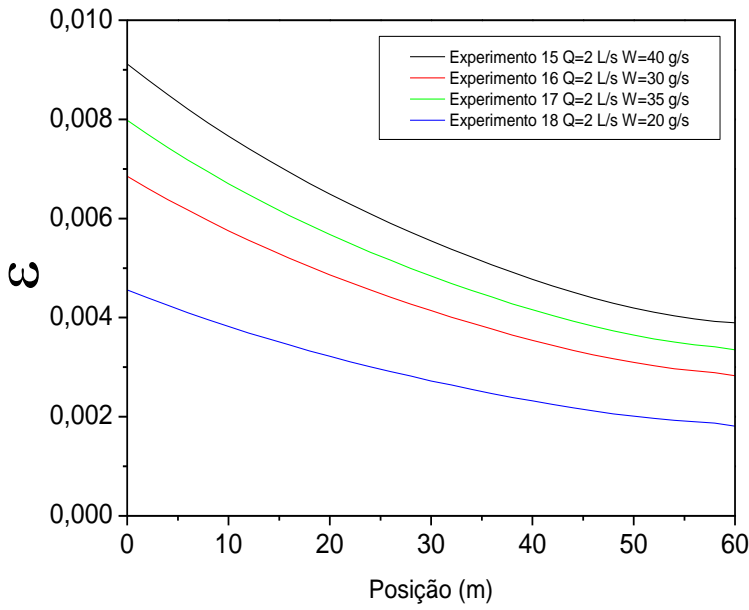

(b)

Figura 4 - Simulações e dados experimentais para concentração em função da posição. Figura 4a dados a $32 \mathrm{~g} / \mathrm{L}$ e Figura $4 \mathrm{~b}$ dados a $258 \mathrm{~g} / \mathrm{L}$.

\section{CONCLUSÕES}

A unidade experimental foi capaz de gerar dados com reprodutibilidade com erros experimentais inferiores a 3\%. Em regime plenamente turbulento e em escoamento em calha foi constatado que o aumento da vazão volumétrica de salmoura alimentada não altera a velocidade de dissolução do sal em salmoura, porém a razão W/Q altera os perfis de dissolução encontrados. O aumento da concentração da fase liquida (salmoura) afeta diretamente a velocidade de dissolução de sal, sendo que soluções mais concentradas tem potencial de dissolução menor. O modelo matemático proposto foi capaz de simular dados experimentais para a faixa trabalhada com erros inferiores a $2,3 \%$. O valor do coeficiente $\mathrm{K}$ foi estimado entre 1,8 e $5,4.10^{-4} \mathrm{~m} / \mathrm{s}$ e correlacionado à concentração da fase líquida. Estes valores foram da mesma ordem de grandeza daquele obtido por Aksel'Rud (1992), 1,0.10 $\mathrm{m} / \mathrm{s}$.

\section{NOMENCLATURA}

$a$ - Área total de transferência de massa

$C^{*}$ - Concentração de saturação

$C$ - Concentração instantânea da solução.

$\mathrm{C}_{\mathrm{o}}$ - Concentração inicial da salmoura

$\overline{\mathrm{D}_{\mathrm{p}}}$ - Diâmetro média de Sauter.

$K$ - Coeficiente convectivo global de transferência de massa

Q - Vazão volumétrica de salmoura

$t-$ Tempo

$\overline{\mathrm{v}}_{\mathrm{Z}}$ - Velocidade média axial de escoamento do fluido

W - Vazão mássica de sal particulado

$X$ - Fração mássica de sal. 
$\rho_{\mathrm{s}}$ - Massa específica do sal

$\varepsilon_{\mathrm{S}}$ - Fração volumétrica de sal na suspensão

$\varepsilon_{\mathrm{o}}$ - Fração volumétrica de sal inicial

\section{REFERÊNCIAS}

ANDERSON, T. F., ABRAMS, D. S. and Grens II, E. A. "Evalutaion of parameters for nonlinear thermodynamic models”, AIChE Journal, vol. 24, No. 1, 1978.

AKSEL'RUD, G.A., BOIKO, A.E. \& KASHCHEEV, A.E. Kinects of the solution of mineral salts suspended in a liquid flow. UDC 532.73-3. SPE (Society of Petroleum Engineers), 1992.

BIRD, R.B., STEWART, W.E. \& LIGHTFOOT, E.N. Transport Phenomena. Second Edition. Chemical Engineering Department. University of Wisconsin-Madison, 2002.

DURIE, R.W \& JESSEN, F.W., Mechanism of the Dissolution of Salt in the Formation of underground Salt Cavities, soc. Pet. Eng. J., spe 678, june 1964, pp. 183-190.

MAGAlHÃES, S. C.; FONSECA, T. F.; DEMAUIR, M.; PINTO, J. C.; CALÇADA, L. A.; SCHEID, C. M. Determinação do Coeficiente de Transferência de Massa do Cloreto de Sódio em Água em Sistemas Agitados. ENEMP, 2011.

MARTINS, L. A. A. "Modelagem e determinação do coeficiente convectivo de transferência de massa de partículas de $\mathrm{NaCl}$ no escoamento de salmoura”. Dissertação de mestrado, PPGEQ, UFRRJ, Rio de Janeiro, Brasil, 2014.

MASSARANI, G., Fluidodinâmica em Sistemas Particulados, Vol. 1, 2ª Edição, 2002.

PATRÃO, W. C. F; "Estudo teórico e experimental da dissolução de partículas de $\mathrm{NaCl}$ no escoamento em salmoura.” Dissertação de mestrado, UFRRJ, Rio de Janeiro, Brasil, 2012. 